\title{
Asymmetric Fabry-Perot Device Arrays with Low Insertion Loss and High Uniformity
}

\author{
A. Jennings, P. Horan, B. Kelly, and J. Hegarty
}

\begin{abstract}
We report on arrays of normally on asymmetric Fabry-Perot modulator (AFPM) devices which exhibit a high degree of uniformity between devices, with a thickness variation across an array of $0.07 \%$. The devices in one array have a mean insertion loss of $1.8 \mathrm{~dB}$, and ofF reflectivities of less than $3 \%$, giving a mean contrast ratio of $22: 1$. These characteristics are measured at a single operating wavelength of $857 \mathrm{~nm}$. The spatial light modulators show promise for the use of AFPM devices in optical processing.
\end{abstract}

$\mathrm{T}_{\mathrm{s}}$ HERE has been considerable interest in developing spatial light modulators which operate with normally incident light because of their potential applications as optical interconnects and for optical computing. Device arrays have been studied using liquid crystals monolithically integrated on CMOS chips [1] with large arrays being demonstrated. These devices have relatively slow switching speeds of $>500 \mu \mathrm{s}$. In III-V quantum-well materials, large arrays of SEED's have been demonstrated [2], which have fast switching times, but rather low contrast ratios.

Until now little attention has been paid to arrays of electrooptic asymmetric Fabry-Perot modulator (AFPM) devices, with papers concentrating on the characteristics of discrete devices. Individual devices have been demonstrated with low operating voltages (less the $3 \mathrm{~V}$ ) [3], high-contrast ratios often in excess of 100:1 [4], or a combination of both low-voltage and high-contrast ratio [5]. This has often been at the expense of insertion loss, with devices having insertion losses in excess of $3 \mathrm{~dB}$. When considering devices for use in optical systems in a cascaded configuration, the insertion loss cannot be neglected. Devices have been reported with low insertion losses of $1.2 \mathrm{~dB}$, but with a poor contrast ratio of 7.5:1 [6] as a result of the relatively high ofF state reflectivity of $10 \%$. Only recently have devices been demonstrated with a combination of a low insertion loss of $1.8 \mathrm{~dB}$, a contrast ratio approaching infinite and low operating voltage of $3.75 \mathrm{~V}$ [7]. Papers on working AFPM devices have ne-

Manuscript received March 25, 1992.

A. Jennings, B. Kelly, and J. Hegarty are with Optronics Ireland Research Centre, Department of Pure and Applied Physics, Trinity College, Dublin, Ireland.

P. Horan is with Hitachi Dublin Laboratory, Trinity College, Dublin, Ireland.

IEEE Log Number 9201821. glected to consider the uniformity of the devices within an array, an issue which must be addressed if AFPM devices are to be considered suitable for use in optical systems. We have succeeded in fabricating arrays of asymmetric Fabry-Perot modulator devices that have been designed for use in a particular optical network [8].

These arrays show excellent uniformity and a very good combination of insertion loss and contrast ratio. This combination of characteristics meets the criterion for application to optical networks.

The problem associated with the uniformity of AFPM devices arises from the fact that small regional fluctuations in the thickness of the wells and barriers of the Fabry-Perot structure will shift the Fabry-Perot resonance wavelength of the device [9]. These small variations do not shift the resonance wavelength of the exciton significantly since the fluctuations in the width of each well or barrier may only be of the order of one monolayer. For instance, a $1 \%$ variation in the thickness of a $1 \mu \mathrm{m}$ thick cavity will shift the operating wavelength of a device designed for 860 by $8.6 \mathrm{~nm}$. The corresponding variation in the resonance wavelength of the excitons in a $10 \mathrm{~nm}$ wide GaAs quantum well will however be less than 0.1 $\mathrm{nm}$.

In the asymmetric Fabry-Perot devices fabricated, the reflectivities of the back and front mirrors are unequal: $R_{b} \approx 95 \%$ and $R_{f} \approx 32 \%$, respectively. With these reflectivities and no intracavity absorption, the reflectivity of the etalon at the Fabry-Perot resonance is approximately $85 \%$. However, by introducing absorption of $\alpha \mathrm{cm}^{-1}$ into the cavity of thickness $d \mathrm{~cm}$ and satisfying the condition: $R_{f}=R_{b} \exp (-2 \alpha d)$, the etalon reflectivity becomes zero at the Fabry-Perot resonance and the cavity is said to be impedance matched. Modulating the absorption of the cavity therefore modulates the etalon reflectivity, providing the basis of an optical modulator with a theoretically infinite contrast ratio and potentially low insertion loss. It is this property of the devices which makes them attractive for use in optical systems.

The asymmetric Fabry-Perot device structure used in this work was grown by atmospheric pressure metalorganic vapor phase epitaxy (MOVPE) on a $n^{+}$GaAs substrate. The structure of the device is as follows. A highly reflective quarter wavelength Bragg mirror containing 15 periods of alternating layers of AlAs (low index) 
and $\mathrm{Al}_{0.1} \mathrm{Ga}_{0.9}$ As (high index), $\mathrm{n}^{+}$doped with Se to $2 \times$ $10^{18} \mathrm{~cm}^{-3}$. This has a reflectivity greater than $95 \%$ at the operating wavelength of $860 \mathrm{~nm}$. A nominally intrinsic MQW structure consisting of 70 periods of $9 \mathrm{~nm} \mathrm{GaAs}$ wells and $6 \mathrm{~nm} \mathrm{Al}{ }_{0.3} \mathrm{Ga}_{0.7}$ As barriers was grown on top of the mirror. The Fabry-Perot cavity was completed by a $0.42 \mu \mathrm{m}$ thick $\mathrm{Al}_{0.3} \mathrm{Ga}_{0.7}$ As layer, $\mathrm{p}^{+}$doped with $\mathrm{Zn}$ to $2 \times 10^{18} \mathrm{~cm}^{-3}$, and a highly doped $50 \AA \mathrm{p}^{+}$GaAs capping layer for contacting purposes.

Arrays of 21 Fabry-Perot modulator devices were fabricated on the grown wafer for use in a specific optical network [8]. The network requires a pattern of finger devices in a linear array. The pattern of this device array is shown in Fig. 1. The active area of each device is $80 \mu \mathrm{m}$ wide and $2.5 \mathrm{~mm}$ long with a $20 \mu \mathrm{m}$ space between each device, giving an active device area in the array of $2.1 \times 2.5$ $\mathrm{mm}$. A $5 \mu \mathrm{m}$ wide electrode runs from the contact pad of the device along the length of the finger to ensure that a uniform voltage is applied along the device length. The electrodes were formed by thermal evaporation of $\mathrm{Au} / \mathrm{Cr}$ metals. The chips were mounted in an DIL IC packages and each device electrically addressed with Au wires ultrasonically bonded to the contact pad.

To characterize each device in the fabricated arrays and monitor the device uniformity, reflectivity spectra of the devices were taken at the corners of the arrays as well as in the centers. These were measured by focusing light from a Ti:sapphire laser onto approximately $20 \mu \mathrm{m}$ diameter spots, scanning the wavelength and measuring the reflected signal. The incident intensity was below $50 \mu \mathrm{W}$ to ensure that no exciton bleaching was taking place in the quantum-well material which would reduce the MQW absorption. Normalization spectra were taken using a high reflecting mirror in place of the device array. Fig. 2 shows reflectivity spectra taken from one array of devices (Array 1) with $0 \mathrm{~V}$ applied to the devices (ON state) and voltages between 10-12 $\mathrm{V}$ applied to the devices (OFF state). A second device array (Array 2) was also characterized showing that the uniformity of the wafer extended beyond the area of Array 1. In Fig. 3, reflectivity spectra of devices from both Arrays 1 and 2 are plotted, with the devices in the ON and OFF states shown. The plot indicates good uniformity between the device arrays, and opens up the possibility of using such devices in optical systems. Both of the arrays were shown to have a combination of low insertion loss, good contrast ratio and high uniformity.

To use arrays of devices in an optical system, all of the devices must be operated at a single wavelength, and so we must concentrate on the characteristics of the devices at a single wavelength. All the devices were therefore characterized at a wavelength of $857 \mathrm{~nm}$, the optimum wavelength for all of the devices on Arrays 1 and 2. A summary of the measured insertion loss, OFF reflectivities, and contrast ratios of all the devices on both device arrays is given in Table I. The results indicate that the devices are compatible for use in an optical network in a cascaded configuration. The characteristics of the device arrays are described below.

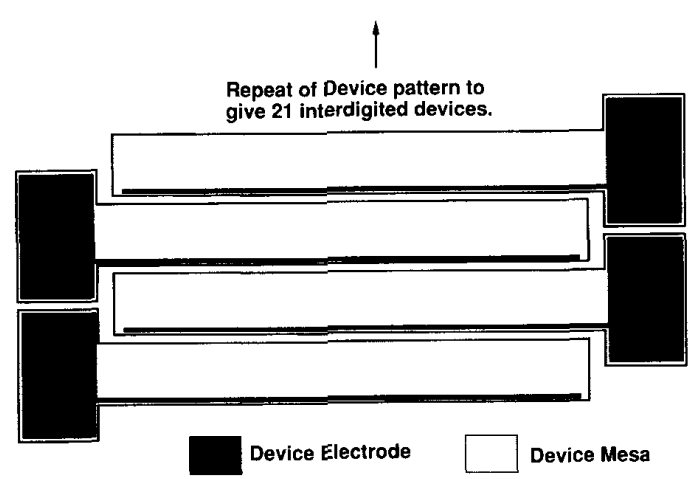

Fig. 1. Device structure of the fabricated arrays of asymmetric Fabry-Perot modulators. The devices fingers are $80 \mu \mathrm{m}$ wide and 2.5 $\mathrm{mm}$ long, with a $20 \mu \mathrm{m}$ spacing between each device, giving a device array area of $2.5 \times 2.1 \mathrm{~mm}$. The $\mathrm{Au} / \mathrm{Cr}$ electrode running along the length of the device is $5 \mu \mathrm{m}$ wide.

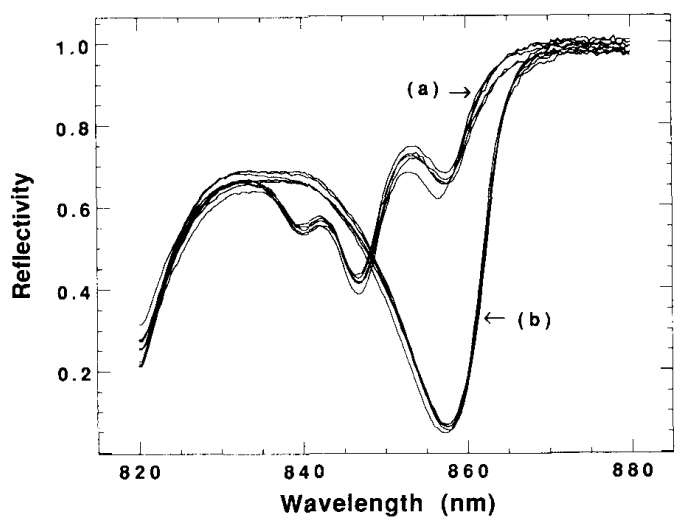

Fig. 2. Reflectivity spectra taken from devices in the fabricated Array 1 . Spectra taken from points in the four corners of the array and in the center. These were taken with $0 \mathrm{~V}$ applied to the devices, shown in the family of plots labeled (a), and with $10 \mathrm{~V}$ applied to the devices, shown in the family of plots labeled (b).

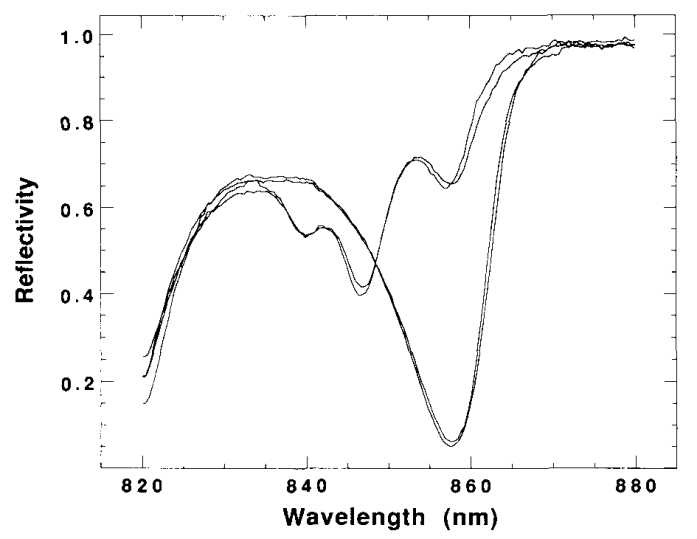

Fig. 3. Reflectivity spectra taken from devices in the fabricated Arrays 1 and 2, with $0 \mathrm{~V}$ and $10 \mathrm{~V}$ applied to the devices for the ON and OFF states, respectively. 
TABLE I

Summary of the Device Characteristics of All tHe Devices in ARRays 1 and 2, AS MEASURed at tHe SINGLE WAVELENGTH OF 857 NM

\begin{tabular}{|c|c|c|c|c|c|c|}
\hline & \multicolumn{2}{|c|}{$\begin{array}{l}\text { Insertion Loss } \\
(\mathrm{dB})\end{array}$} & \multicolumn{2}{|c|}{$\begin{array}{l}\text { OFF Reflectivities } \\
(\%)\end{array}$} & \multicolumn{2}{|c|}{ Contrast Ratio } \\
\hline & Mean & $\begin{array}{c}\text { Mean } \\
\text { Deviation }\end{array}$ & Mean & $\begin{array}{c}\text { Mean } \\
\text { Deviation }\end{array}$ & Mean & $\begin{array}{c}\text { Mean } \\
\text { Deviation }\end{array}$ \\
\hline & 2.1 & 0.1 & 3.2 & 0. & 19.4 & 1. \\
\hline Array 2 & 1.8 & 0.1 & 3.0 & 0.3 & 21.8 & 1.8 \\
\hline
\end{tabular}

All of the devices on Array 1 were characterized and shown to have an average insertion loss of $2.0 \mathrm{~dB}$ ranging from 1.8 to $2.2 \mathrm{~dB}$. The average ofF reflectivity measured for the devices was $3.2 \%$, ranging from $2.8 \%$ to $3.8 \%$ and the average contrast ratio was 19:1. The characteristics of Array 2 showed an improvement on Array 1, with an average insertion loss in the devices of $1.8 \mathrm{~dB}$, with insertion losses ranging from 1.7 to $2.0 \mathrm{~dB}$. The reflectivity of the devices in the OFF state was in the range of $2.7 \%$ to $3.8 \%$, with a mean value of $3.0 \%$, and the average contrast ratio was $22: 1$, with values ranging from $18: 1$ to $24: 1$. The wavelength of the Fabry-Perot resonance, as measured across the device array, shifted from 856.8 to 857.4 $\mathrm{nm}$, giving a thickness uniformity of $0.07 \%$ within the area of the array. This is equivalent to a variation in the thickness of the device structure of approximately $1 \mathrm{~nm}$.

For the application of devices in optical networks, we must consider the combination of the device characteristics of insertion loss and contrast ratio together, rather than individually. Once the OFF reflectivity approaches about $1 \%$, then getting to lower reflectivities becomes less crucial. The next important criterion to consider is the oN reflectivity, or insertion loss of the devices, which determines the extent to which the devices can be used in a cascaded configuration in an optical network. The combination of an insertion loss of $1.8 \mathrm{~dB}$ and OFF reflectivity of $3 \%$, with consequent contrast ratios of $22: 1$, achieved in these devices indicates the devices are useful from a systems point of view. Other device types demonstrating contrast ratios of $20: 1$ have been successfully implemented in optical neural network systems [10]. The achievement of such characteristics over the whole array of AFPM devices within a very tight tolerance opens the possibility for the integration of these devices in optical systems.
In conclusion, we have demonstrated large area arrays of AFPM devices with a uniformity within $0.07 \%$. The mean insertion loss of the devices on one array was measured to be $1.8 \mathrm{~dB}$, and the reflectivity of the devices was modulated down to $3 \%$, giving an average contrast ratio of 22:1. The problem of uniformity in arrays of AFPM devices is a problem that must be overcome if these devices are to be considered suitable for use in optical systems. We believe that the arrays of devices reported have uniformities and operating characteristics which make them suitable for use in optical networks.

\section{ACKNOWLEDGMENT}

We would like to thank J. S. Roberts at the SERC III-V Semiconductor Facility, University of Sheffield, UK, for growing the wafer used to fabricate the device arrays.

\section{REFERENCES}

[1] D. A. Jared and K. M. Johnson, "Optically addressable thresholding very-large-scale-integration/liquid-crystal spatial light modulators," Opt. Lett., vol. 16, pp. 967-969, 1991.

[2] L. M. F. Chirovski, L.A. D'Asaro, J. M. Freund, J. M. Kopf, A. L. Lentine, and T. Sizer, "Improving the performance of large arrays of Symmetric Self Electrooptic Effect Devices," Tech. Dig., Photon. Switching, (Kobe, Japan), p. 243, 1990.

[3] R. H. Yan, R. J. Simes, L. A. Coldren, and A. C. Gossard, "Transverse modulators with a record reflection change of > 20\%V using asymmetric Fabry-Perot structures," Appl. Phys. Lett., vol. 56, pp. 1626-1628, 1990.

[4] M. Whitehead, A. Rivers, and G. Parry, "Low voltage multiple quantum well reflection modulator with ON:OFF ratio > 100:1," Electron. Lett., vol. 25, pp. 984-985, 1989.

[5] K. K. Law, R. H. Yan, L. A. Coldren, and J. L. Merz, "Self-electrooptic device based on a superlattice asymmetric Fabry-Perot modulator with an ON/OFF ratio >100:1," Appl. Phys. Lett., vol. 57, pp. $1345-1347,1990$.

[6] B. Pezeshki, D. Thomas, and J. S. Harris, Jr., "Optimization of modulation ratio and insertion loss in reflective electroabsorption modulators," Appl. Phys. Lett., vol. 57, pp. 1491-1493, 1990.

[7] K.-K. Law, M. Whitehead, J. L. Merz, and L. A. Coldren, "Simultaneous achievement of low insertion loss, high contrast and low operating voltage in asymmetric Fabry-Perot reflection modulator," Electron. Lett., vol. 27, pp. 1863-1865, 1991.

[8] P. Horan, D. Uecker, and A. Arimoto, "Optical implementation of a second-order neural network discriminator model," Jap. J. Appl. Phys., vol. 29, pp. L1328-L1331, 1990.

[9] G. Parry, M. Whitehead, P. Zouaneli, A. Rivers, K. Woodbridge, J. S. Roberts, and C. Button, "Some practical issues associated with the design and fabrication of high contrast quantum well modulator arrays," Tech. Dig. Opt. Computing, Salt Lake City, UT, OSA Tech. Dig., vol. 61, MD1 pp. 1-4, 1991.

[10] L. Zhang, M. G. Robinson, and K. M. Johnson, "Optical implementation of a second-order neural network," Opt. Lett., vol. 16, pp. 45-47, 1991 